\title{
Cosmological Implications of the CMB Large-scale Structure
}

\author{
Fulvio Melia ${ }^{1}$ \\ Department of Physics, The Applied Math Program, and Department of Astronomy, \\ The University of Arizona, AZ 85721, USA \\ fmelia@email.arizona.edu
}

Received

accepted 


\begin{abstract}
The Wilkinson Microwave Anisotropy Probe (WMAP) and Planck may have uncovered several anomalies in the full cosmic microwave background (CMB) sky that could indicate possible new physics driving the growth of density fluctuations in the early Universe. These include an unusually low power at the largest scales and an apparent alignment of the quadrupole and octopole moments. In a $\Lambda$ CDM model where the CMB is described by a Gaussian Random Field, the quadrupole and octopole moments should be statistically independent. The emergence of these low probability features may simply be due to posterior selections from many such possible effects, whose occurrence would therefore not be as unlikely as one might naively infer. If this is not the case, however, and if these features are not due to effects such as foreground contamination, their combined statistical significance would be equal to the product of their individual significances. In the absence of such extraneous factors, and ignoring the biasing due to posterior selection, the missing large-angle correlations would have a probability as low as $\sim 0.1 \%$ and the low- $l$ multipole alignment would be unlikely at the $\sim 4.9 \%$ level; under the least favourable conditions, their simultaneous observation in the context of the standard model could then be likely at only the $\sim 0.005 \%$ level. In this paper, we explore the possibility that these features are indeed anomalous, and show that the corresponding probability of CMB multipole alignment in the $R_{\mathrm{h}}=c t$ Universe would then be $\sim 7-10 \%$, depending on the number of large-scale SachsWolfe induced fluctuations. Since the low power at the largest spatial scales is reproduced in this cosmology without the need to invoke cosmic variance, the overall likelihood of observing both of these features in the CMB is $\geq 7 \%$,
\end{abstract}


much more likely than in $\Lambda$ CDM, if the anomalies are real. The key physical ingredient responsible for this difference is the existence in the former of a maximum fluctuation size at the time of recombination, which is absent in the latter because of inflation.

Subject headings: cosmic background radiation; cosmology: theory; early universe; gravitation; inflation 


\section{Introduction}

The Wilkinson Microwave Anisotropy Probe (WMAP) and the Planck satellite have revolutionized our ability to study anisotropies in the cosmic microwave background (CMB) with a precision that is now permitting us to examine the structure of the Universe on all scales (Bennett et al. 2003; Ade et al. 2013). But several apparent anomalies may be indicating possible new physics driving the origin of density fluctuations in the early Universe and their evolution into the large-scale structure we see today. These peculiarities include an unusually low power at the largest scales (Spergel et al. 2003), as well as an apparent alignment of the quadrupole and octopole moments (Tegmark et al. 2003; de Oliveira-Costa et al. 2004; Hansen et al. 2004; Eriksen et al. 2004; Schwarz et al. 2004; Land \& Magueijo 2005). Prior to the Planck era, these features had variously been attributed to astrophysical, instrumental, or cosmological causes, and even faulty data analysis or a posteriori statistics (Copi et al. 2009). The possibility that these features might be due to instrumental effects, however, has recently been weakened by the Planck mission, which has confirmed the low power on the largest scales and an alignment between $9^{\circ}$ and $13^{\circ}$ of the quadrupole and octopole orientations (Ade et al. 2013). The low power and alignment are puzzling because the probability of either occurring within the context of the standard model $(\Lambda \mathrm{CDM})$, under the assumption that neither is the result of mere posterior selection biases (Bennett et al. 2011), is less than $\sim 1 \%$; the chance to measure the sky with both has been quantified at $<10^{-6}$ (Sarkar et al. 2011).

The power on large scales is characterized in terms of the angular correlation function $C(\theta)$ (defined in Eq. 2 below). According to the observations, $C(\theta)$ essentially vanishes at angular separations greater than about $60^{\circ}$ (Spergel et al. 2003; Ade et al. 2013), confirming what was first measured a decade earlier with the Cosmic Background Explorer

\footnotetext{
${ }^{1}$ John Woodruff Simpson Fellow.
} 
(COBE; Hinshaw et al. 1996; Wright et al. 1996). The absence of any angular correlation at the largest scales could be a problem for the standard model because it disagrees with the expectations of an inflationary scenario (Guth 1981; Linde 1982). Yet without inflation, $\Lambda \mathrm{CDM}$ could not account for the apparent uniformity of the CMB (other than the aforementioned anisotropies at the level of 1 part in 100,000) across the sky.

But though the reality of these anomalies is no longer questioned, the possible reasons for their existence, and their significance, are still being discussed and evaluated. As Copi et al. (2010) have pointed out in their review on this subject, four classes of explanations have thus far been proposed, including astrophysical causes, such as foreground effects, faulty data analysis, instrumental systematics, and perhaps cosmological reasons. One can find faults with each of these explanations, so no consensus has yet been reached on which is the most likely to account for the observations. Nonetheless, an obvious possible cause of the anisotropy is contamination by a pernicious foreground (see, e.g., Slosar and Seljak 2004; Bielewicz et al. 2005; Copi et al. 2006). In this class of explanations, some workers have suggested that the observed quadrupole-octopole alignment might be due to the Rees-Sciama effect (Rakic et al. 2006; Rakic and Schwarz 2007), interstellar dust (Frisch 2005), the presence of local voids (Inoue and Silk 2006), or even the Sunyaev-Zeldovich effect (Peiris and Smith 2010). Another proposal by Vale (2005) argues that the unexpected anisotropy might be due to a moving lens effect associated with the Great Attractor, though its influence may be too small to fully account for the observations (Cooray and Seto 2005). And on a more local scale, Dikarev et al. (2008,2009) have argued that the solar system dust could give rise to sizable levels of microwave emission and absorption.

Artifacts produced by faulty data analysis could also produce unexpected anisotropies. When using reconstructed full-sky maps (e.g., Bennett et al. 2003; Tegmark et al. 2003; Eriksen et al. 2004), a sky cut of just a few degrees produces errors in the reconstructed 
anisotropy pattern and the directions of multipole vectors that are too large to allow quantitative conclusions concerning the apparent alignments (Copi et al. 2004). Efstathiou et al. (2010) have argued that one should instead use maximum likelihood estimators to the cut-sky maps to reliably reconstruct the CMB anisotropy distribution. They argue that estimating the probability of seeing these anomalies based on the pixel approach using the cut sky is simply a coincidence, and that a more reliable result is produced from their maximum-likelihood reconstruction technique.

Prior to the observations by Planck, which largely confirmed the reality of the CMB anomalies, instrumental explanations also played a role. These are perhaps not as likely now that WMAP and Planck confirm each other's measurements, though one can not be sure that all systematics have been eliminated (Bennett et al. 2003). For example, imperfections in the instrument may couple with dominant signals from the sky to create anomalies (Gordon et al. 2005).

The WMAP team itself considers the CMB anomalies, such as the low-multipole alignment, to be real, though they question the significance of these results and the possibility that they may be due to cosmological influences (Bennett et al. 2011). They note that Chiang et al. (2007) find that the lowest spherical harmonic modes in the ILC map are significantly contaminated by foregrounds. And Park et al. (2007) conclude that the residual foreground emission based on their own analysis is not statistically important to the large-scale modes of the CMB. Bennet et al. (2011) also support the view that instead of early universe effects, the apparent low-multipole alignment may be due to the integrated Sachs-Wolfe (ISW) effect associated the local $(z<0.3)$ density fields. Francis and Peacock (2010) estimated this density field from the 2MASS and SuperCOSMOS galaxy catalogs and used these to calculated the ISW effect within this volume. When they removed their estimated ISW contribution from the WMAP map, the quadrupole 
amplitude increased while that of the octopole remained relatively unchanged. More importantely, they concluded that no significant quadrupole-octopole alignment remained after this subtraction.

One therefore must be wary about placing too much confidence in the notion that the low-multipole alignment is necessarily a signature of cosmological effects in the early Universe. However, the origin of this alignment, which is generally considered to be real, is still unknown. In this paper, we consider whether a cosmological basis for this low-l multipole alignment could be used to distinguish between the $\Lambda$ CDM and $R_{\mathrm{h}}=c t$ expansion scenarios. Specifically, we seek to address the question of whether an alignment that may be statistically unexpected in the context of the standard model could instead be more in line with that expected in the $R_{\mathrm{h}}=c t$ Universe (Melia 2007; Melia \& Shevchuk 2012; Melia 2013a) which, unlike $\Lambda$ CDM, did not undergo a period of early inflation. Earlier, we showed that the absence of power on large scales exhibited by the angular correlation function might be evidence in support of this model simply because it does not require inflation (Melia 2014). Here, we discuss whether an absence of early inflation might also provide an explanation for the apparent alignment of the CMB's quadrupole and octopole moments. We will consider the conditions under which large-scale fluctuations in the $R_{\mathrm{h}}=c t$ cosmology could account for the low- $l$ portion of the spectrum. As we shall see, the constraints emerging from this analysis will allow us to determine the kinds of all-sky map one would associate with the $R_{\mathrm{h}}=c t$ condition. We will carry out a statistical analysis of thousands of simulated renderings to calculate the probability of seeing an apparent alignment of the CMB quadrupole and octopole moments in the $R_{\mathrm{h}}=c t$ Universe, and compare this with that expected in $\Lambda$ CDM.

Since this is the first attempt at simulating the fluctuation spectrum in $R_{\mathrm{h}}=c t$, we will necessarily restrict our attention to the influence most responsible for producing the 
quadrupole and octopole moments - the Sachs-Wolfe effect (Sachs \& Wolfe 1967). Other phenomena, such as the Baryon Acoustic Oscillations, influence primarily the fluctuation growth on sub-degree scales. We will incorporate these effects in future applications of this work, but for the sake of keeping the interpretation of the results as simple as possible, focusing primarily on the largest fluctuations, we will not include them here.

\section{The CMB Angular Power Spectrum and Correlation Function}

The CMB temperature anisotropies $\Delta T(\Omega) / T$ extracted from WMAP and Planck may be written as an expansion using spherical harmonics $Y_{l m}(\hat{\mathbf{n}})$,

$$
\frac{\Delta T(\Omega)}{T}=\sum_{l m} a_{l m} Y_{l m}
$$

from which one can then determine the two-point angular correlation function (for directions $\hat{\mathbf{n}}_{1}$ and $\left.\hat{\mathbf{n}}_{2}\right)$ :

$$
C(\cos \theta) \equiv\left\langle T\left(\hat{\mathbf{n}}_{1}\right) T\left(\hat{\mathbf{n}}_{2}\right)\right\rangle=\frac{1}{4 \pi} \sum_{l}(2 l+1) C_{l} P_{l}(\cos \theta) .
$$

Statistical independence implies that

$$
\left\langle a_{l m}^{*} a_{l^{\prime} m^{\prime}}\right\rangle \propto \delta_{l l^{\prime}} \delta m m^{\prime}
$$

and statistical isotropy further requires that the constant of proportionality depend only on $l$, not $m$ :

$$
\left\langle a_{l m}^{*} a_{l^{\prime} m^{\prime}}\right\rangle=C_{l} \delta_{l l^{\prime}} \delta m m^{\prime}
$$

The constant

$$
C_{l}=\frac{1}{2 l+1} \sum_{m}\left|a_{l m}\right|^{2}
$$

is the angular power of the multipole $l$.

A comparison of the function $C(\theta)$ predicted by the $R_{\mathrm{h}}=c t$ Universe with that observed by WMAP was the primary goal of our previous paper (Melia 2014). Our focus 
here will be the second CMB anomaly discussed above, i.e., the apparent alignment of $C_{2}$ and $C_{3}$. To quantify the statistical significance of this alignment, we will follow the procedure developed by (de Oliveira-Costa et al. 2004). Other techniques have been utilized since then (Copi et al. 2009), but they all appear to confirm each other's results, so for now, at least, we will base our assessment on the former approach.

The method treats the CMB map as a wave function,

$$
\frac{\Delta T}{T}(\hat{\mathbf{n}}) \equiv \psi(\hat{\mathbf{n}})
$$

and seeks to find the axis $\hat{\mathbf{n}}$ about which the "angular momentum" dispersion

$$
\left\langle\psi\left|(\hat{\mathbf{n}} \cdot \mathbf{L})^{2}\right| \psi\right\rangle=\sum_{m} m^{2}\left|a_{l m}(\hat{\mathbf{n}})\right|^{2}
$$

is maximized. The coefficients $a_{l m}(\hat{\mathbf{n}})$ correspond to the spherical harmonics in a rotated coordinate system with the $z$-axis in the $\hat{\mathbf{n}}$ direction. For the actual CMB map, the maximization is performed by evaluating Eq. (7) at all the unit vectors $\hat{\mathbf{n}}$ corresponding to the pixel centers. We will follow essentially the same approach, first producing a rendering of the large-scale fluctuations on the whole sky, and then maximizing the angular momentum dispersion using the same equation (more on this below). Our synthetic images each contain $180 \times 360$ pixels in $\theta$ and $\phi$, respectively.

Previous papers have reported the preferred axes $\hat{\mathbf{n}}_{2}$ and $\hat{\mathbf{n}}_{3}$ for the quadrupole and octopole moments to be

$$
\begin{aligned}
& \hat{\mathbf{n}}_{2} \sim(-0.1145,-0.5265,0.8424) \\
& \hat{\mathbf{n}}_{3} \sim(-0.2578,-0.4207,0.8698)
\end{aligned}
$$

respectively, i.e., both roughly in the direction $(l, b) \sim\left(-110^{\circ}, 60^{\circ}\right)$ in Virgo (de Oliveira et al. 2004). In $\Lambda \mathrm{CDM}$, a crucial ingredient is cosmological inflation - a brief phase of very rapid expansion from approximately $10^{-35}$ seconds to $10^{-32}$ seconds following the big 
bang, forcing the Universe to expand much more rapidly than would otherwise have been feasible solely under the influence of matter, radiation, and dark energy. This accelerated expansion would have driven the growth of fluctuations on all scales, resulting in an angular correlation at all angles (which does not appear to be consistent with the WMAP and Planck results; Melia 2014). Therefore, in $\Lambda$ CDM, the unit vectors $\hat{\mathbf{n}}_{2}$ and $\hat{\mathbf{n}}_{3}$ should be independently drawn from a distribution in which all directions are equally likely. This means that the dot product $\hat{\mathbf{n}}_{2} \cdot \hat{\mathbf{n}}_{3}$ should be a uniformly distributed random variable on the interval $(-1,1)$. But as is well known, Eq. (7) does not distinguish between $\hat{\mathbf{n}}$ and $-\hat{\mathbf{n}}$, so the maximization procedure finds a preferred axis, not a preferred direction. The alignment should therefore be quantified on the basis of $\left|\hat{\mathbf{n}}_{2} \cdot \hat{\mathbf{n}}_{3}\right|$, which instead has a uniform distribution on the interval $(0,1)$ (de Oliveira-Costa et al. 2004).

The anomaly emerges when we determine from Eq. (8) that the observed value of this dot product is $\left|\hat{\mathbf{n}}_{2} \cdot \hat{\mathbf{n}}_{3}\right| \approx 0.9838$, corresponding to a separation of only $10.3^{\circ}$. An alignment this good happens by chance only once in 62 realizations, suggesting that the probability of finding a random octopole axis within a circle of radius $10.3^{\circ}$ of the quadrupole axis should be less than a few percent. Within the context of $\Lambda \mathrm{CDM}$, this alignment is therefore a statistically significant anomaly, in terms of the standard definition in which an outcome with probability $<5 \%$ is considered to be significantly anomalous. (Note, however, that this may still be $<3 \sigma$.)

Recognizing that producing an accurate map of the WMAP data depends critically on correctly identifying the background (or more accurately in this case, the foreground), this calculation has been repeated on several occasions, with an ever increasing precision of the foreground subtraction. The numbers themselves have changed somewhat, but all subsequent measurements have confirmed the early conclusions. The most likely outcome currently appears 
to be an alignment angle $3.8^{\circ}<\theta_{23}<18.2^{\circ}$ (Park et al. 2007). Even with such a broadened uncertainty range, however, an alignment within $\theta_{23} \sim 18^{\circ}$ should occur only $\sim 4.9 \%$ of the time, making it a marginally statistically significant anomaly within the standard model. And as we noted earlier, the latest results from the Planck mission appear to confirm these earlier conclusions, though with a somewhat different range of possible angles, $\sim 9^{\circ}-13^{\circ}$. The implied alignment for both WMAP and Planck sits comfortably within the interval $\theta_{23} \leq 18^{\circ}$, and we will therefore use this conservatively large range for all of the analysis that follows.

3. Large-Scale Fluctuations in the $R_{\mathrm{h}}=c t$ Universe

The $R_{\mathrm{h}}=c t$ Universel is an FRW cosmology (Melia 2007; Melia \& Shevchuk 2012) that adheres very closely to the restrictions imposed on the theory by the Cosmological Principle and the Weyl postulate (Weyl 1923). Taken seriously, these two basic tenets force the gravitational horizon $R_{\mathrm{h}}$ (recognized more commonly as the Hubble radius) to always equal $c t$, where $t$ is the cosmic time. It is easy to convince oneself that this equality forces the expansion rate to be constant, so the expansion factor $a(t)$ appearing in the Friedmann equations must be $t / t_{0}$ (utilizing the convention that $a\left(t_{0}\right)=1$ today), where $t_{0}$ is the current age of the Universe.

$\Lambda \mathrm{CDM}$ appears to be an approximation to this cosmology because it adopts a specific set of constituents for the energy density $\rho$, though without the important constraint that the overall equation of state must be $p=-\rho / 3$, where $p$ and $\rho$ are the total pressure and density, respectively. Therefore, $R_{\mathrm{h}}$ in $\Lambda$ CDM fluctuates about the mean it would otherwise always have, leading to the 
awkward situation in which the value of $R_{\mathrm{h}}\left(t_{0}\right)$ is equal to $c t_{0}$ today, but in order to achieve this "coincidence", the Universe had to decelerate early on, followed by a more recent acceleration that exactly balanced out the effects of its slowing down at the beginning. It is specifically this early deceleration in $\Lambda$ CDM that brings it into conflict with the near uniformity of the CMB data, requiring the introduction of an inflationary phase to rescue it. As shown in Melia (2014), however, the recent assessment of the observed angular correlation function suggests that a possible reason for the lack of correlation at large angles may be the absence of an inflationary episode.

Over the past several years, we have tested the predictions of $R_{\mathrm{h}}=c t$ against several different types of observational data, both at low and high redshifts. These efforts have demonstrated that, statistically speaking, the $R_{\mathrm{h}}=c t$ Universe is more likely than $\Lambda$ CDM to be the correct description of nature (see, e.g., Melia \& Maier 2013; Melia 2013b; Wei et al. 2013). In this paper, we add to the comparative study of $\Lambda$ CDM versus $R_{\mathrm{h}}=c t$, by examining how the large-scale fluctuations in these two cosmologies account for the CMB data, focusing on the question of whether the near alignment of the quadrupole and octopole moments ought to be viewed as statistically significant.

As shown in Melia \& Shevchuk (2012) and Melia (2014), density fluctuations $\delta \equiv \delta \rho / \rho$, written as a wavelike decomposition

$$
\delta=\sum_{\kappa} \delta_{\kappa}(t) e^{i \vec{\kappa} \cdot \mathbf{r}}
$$

satisfy the differential equation

$$
\ddot{\delta}_{\kappa}+\frac{3}{t} \dot{\delta}_{\kappa}=\frac{1}{3} c^{2}\left(\frac{\kappa}{a}\right)^{2} \delta_{\kappa}
$$

The way perturbation growth is handled in $R_{\mathrm{h}}=c t$, leading to Equation (10), is somewhat different from $\Lambda \mathrm{CDM}$, so let's take a moment to briefly describe the origin of this expression. 
The chief difference between $\Lambda \mathrm{CDM}$ and $R_{\mathrm{h}}=c t$ is that one must guess the constituents of $\rho$ in the former, assign an individual equation of state to each, and then solve the growth equation derived for each of these components separately. This is how one handles a situation in which the various species do not necessarily feel each other's pressure, though they do feel the gravitational influence from the total density. The coupled equations of growth for the various components can be quite complex, so one typically approximates the equations by expressions that highlight the dominant species in any given era. For example, before recombination, the baryon and photon components must be treated as a single fluid, since they are coupled by frequent interactions in an optically-thick environment. During this period, $\Lambda \mathrm{CDM}$ assumes that "dark energy" is smooth on scales corresponding to the fluctuation growth, and treats the baryon-photon fluid as a single perturbed entity with the pressure of radiation and an overall energy density corresponding to their sum. Once the radiation decouples from the luminous matter, all four constituents (including dark matter) must be handled separately.

The situation in $R_{\mathrm{h}}=c t$ is quite different for several reasons. First of all, the overall equation of state in this cosmology is not forced on the system by the constituents; it is the other way around. The symmetries implied by the Cosmological Principle and Weyl's postulate together, through the application of general relativity, only permit a constant expansion rate, which means that $p=-\rho / 3$. The expansion rate depends on the total energy density, but not on the partitioning among the various constituents. Instead, the constituents must partition themselves in such a way as to always guarantee that this overall equation of state is maintained during the expansion.

And since the pressure is therefore a non-negligible fraction of $\rho$ at all times, one cannot use the equations of growth derived from Newtonian theory (commonly employed in $\Lambda \mathrm{CDM})$, since $p$ itself acts a source of curvature. One must therefore necessarily start with 
the relativistic growth equation (numbered 41 in Melia \& Shevchuk 2012), which correctly incorporates all of the contributions from $\rho$ and $p$. This equation is ultimately derived from Einstein's field equations using the perfect-fluid form of the stress-energy tensor, written in terms of the total $\rho$ and total $p$, but without specifying the sub-partitioning of the density and pressure among the various constituents. With this approach, there is only one growth equation.

On occasion, it is also necessary to use the relativistic growth equation in $\Lambda$ CDM. But there, one typically chooses a regime where a single constituent is dominant, say during the matter-dominated era, and then one assumes that $\rho$ is essentially just the density due to matter (for which also $p \approx 0$ ). But in general, since the pressure appearing in the stress-energy tensor is the total pressure, one cannot mix and match different components that may or may not "feel" each other's influence (as described above). So in fact using the relativistically correct growth equation is difficult in $\Lambda$ CDM, unless one can make suitable approximations in a given regime.

In $R_{\mathrm{h}}=c t$, on the other hand, the total pressure is always $-\rho / 3$, so the key question is whether all of the constituents participate in the perturbation growth, or whether only some of them do. There is no doubt that the baryons and photons are coupled prior to recombination. In $\Lambda \mathrm{CDM}$, one assumes that dark energy is coupled only weakly, acting as a smooth background. In $R_{\mathrm{h}}=c t$, dark energy cannot be a cosmological constant. One therefore assumes that during the early fluctuation growth, everything is coupled strongly in order to maintain the required total pressure $-\rho / 3$. This may change locally once the matter has clumped if it decouples from dark energy on such small scales.

In short, there is one assumption made in each cosmology. In $\Lambda \mathrm{CDM}$, dark energy is a cosmological constant that remains smooth while the baryon-photon fluid is perturbed at early times. In $R_{\mathrm{h}}=c t$, dark energy cannot be a cosmological constant, and everything is 
coupled strongly at early times, so the perturbation affects the total energy density $\rho$. One must always use the correct relativistic growth equation, which includes $p$ as a source of gravity.

In the end, this equation simplifies considerably because the active mass in $R_{\mathrm{h}}=c t$ is proportional to $\rho+3 p=0$, and therefore the gravitational term normally appearing in the standard model is absent. But this does not mean that $\delta_{\kappa}$ cannot grow. Instead, because $p<0$, the (usually dissipative) pressure term on the right-hand-side here becomes an agent of growth. Moreover, there is no Jeans length scale. In its place is the gravitational radius, which we can see most easily by recasting this differential equation in the form

$$
\ddot{\delta}_{\kappa}+\frac{3}{t} \dot{\delta}_{\kappa}-\frac{1}{3} \frac{\Delta_{\kappa}^{2}}{t^{2}} \delta_{\kappa}=0
$$

where

$$
\Delta_{\kappa} \equiv \frac{2 \pi R_{\mathrm{h}}}{\lambda}
$$

Note, in particular, that both the gravitational radius $R_{\mathrm{h}}$ and the fluctuation scale $\lambda$ vary with $t$ in exactly the same way, so $\Delta_{\kappa}$ is therefore a constant in time. But the growth rate of $\delta_{\kappa}$ depends critically on whether $\lambda$ is less than or greater than $2 \pi R_{\mathrm{h}}$.

A simple solution to equation (11) is the power law

$$
\delta_{\kappa}(t)=\delta_{\kappa}(0) t^{\alpha}
$$

where

$$
\alpha^{2}+2 \alpha-\frac{1}{3} \Delta_{\kappa}^{2}=0
$$

That is,

$$
\alpha=-1 \pm \sqrt{1+\Delta_{\kappa}^{2} / 3}
$$

so for small fluctuations ( $\lambda<<2 \pi R_{\mathrm{h}}$ ), the growing mode is

$$
\delta_{\kappa} \sim \delta_{\kappa}(0) t^{\Delta_{\kappa} / \sqrt{3}}
$$


whereas for large fluctuations $\left(\lambda>2 \pi R_{\mathrm{h}}\right)$, the dominant mode

$$
\delta_{\kappa} \sim \delta_{\kappa}(0)
$$

does not even grow. The second mode decays away for both small and large fluctuations.

Insofar as the quadrupole and octopole moments are concerned, the most critical aspect of the fluctuations implied by these equations is the maximum range over which they would have grown. The required inflated expansion in $\Lambda$ CDM drives the growth over all scales. In the $R_{\mathrm{h}}=c t$ Universe, on the other hand, the growth is limited to a maximum fluctuation size

$$
\lambda_{\max }(t) \sim 2 \pi R_{\mathrm{h}}(t)
$$

Thus, since the comoving distance to the last scattering surface (at time $t_{e}$ ) is

$$
r_{e}=c t_{0} \int_{t_{e}}^{t_{0}} \frac{d t^{\prime}}{t^{\prime}}=c t_{0} \ln \left(\frac{t_{0}}{t_{e}}\right)
$$

the maximum angular size $\theta_{\max }$ of any fluctuation associated with the CMB emitted at $t_{e}$ has to be

$$
\theta_{\max }=\frac{\lambda_{\max }\left(t_{e}\right)}{R_{e}\left(t_{e}\right)}
$$

where

$$
R_{e}\left(t_{e}\right)=a\left(t_{e}\right) r_{e}=a\left(t_{e}\right) c t_{0} \ln \left(\frac{t_{0}}{t_{e}}\right)=c t_{e} \ln \left(\frac{t_{0}}{t_{e}}\right)
$$

is the proper distance to the last scattering surface at time $t_{e}$. That is,

$$
\theta_{\max } \sim \frac{2 \pi}{\ln \left(t_{0} / t_{e}\right)}
$$

For the sake of illustration, we note that the times $t_{0}=13.7$ Gyr and $t_{e} \approx 380,000$ yrs from the standard model would imply $\theta_{\max } \sim 34^{\circ}$. It is the existence of this limit that allows the $R_{\mathrm{h}}=c t$ Universe to fit the angular correlation function much better than $\Lambda \mathrm{CDM}$, and we shall see shortly that the existence of this limit also alters the probability of seeing a low-multipole alignment of the CMB, rendering it statistically insignificant. 
In the spirit of identifying the key elements of the theory responsible for the CMB fluctuations, without necessarily getting lost in the details of the complex treatment involving fluctuation growth on small and large scales, and the impact of transfer functions that link the observed temperature variations to the incipient density perturbations, we will here follow the same approach described in (Melia 2014), which itself is based on simplified methods used in earlier applications (Efstathiou 1990).

The Sachs-Wolfe effect dominates the fluctuation growth on scales larger than $\sim 1^{\circ}$ (Sachs \& Wolfe 1967). In $\Lambda$ CDM, the assumption is now made that by the time these fluctuations have formed, one can ignore the contribution of radiation pressure to the total active mass in the fluid, so that at these large wavelengths, the amplitude of the temperature fluctuation ought to scale solely with the local gravitational potential (Efstathiou 1990). For this to be valid, one must also assume that dark energy (presumably a cosmological constant) functions as a smooth background. In $R_{\mathrm{h}}=c t$, the corresponding sequence of steps is similar, with analogous assumptions concerning the behavior of dark energy (which in this case cannot be a cosmological constant). In this model, the zero active mass condition, $\rho+3 p=0$, applies on large scales, where the (Hubble) flow is relatively smooth, but not necessarily on small scales once the matter has clumped. At the beginning when the fluctuations start to grow, all the constituents, including matter, radiation, and dark energy, are coupled together and produce a total pressure $p=-\rho / 3$. This is reflected in the form of Equation (11) and its solutions. But as in $\Lambda$ CDM, the assumption is made here that dark energy does not clump on scales comparable to matter. Thus, while matter still "feels" the total pressure (due to radiation and dark energy), this pressure is relatively uniform, and therefore does not contribute to the local potential. 
We therefore assume that on this scale (as opposed to the larger, smooth Hubble flow), only the gravitational potential associated with the energy density $\rho$ influences the CMB. It is therefore not difficult to show that

$$
\frac{\Delta T}{T} \sim \delta \rho \lambda^{2}
$$

The variance in density over a particular comoving scale $\lambda$ is given as

$$
\left(\frac{\delta \rho}{\rho}\right)_{\lambda}^{2} \propto \int_{0}^{\kappa \sim 1 / \lambda} P\left(\kappa^{\prime}\right) d^{3} \kappa^{\prime}
$$

(see, e.g., Efstathiou 1990), where $P(\kappa)=\left\langle\left|\delta_{\kappa}\right|^{2}\right\rangle$ is the power spectrum. Not knowing the exact form of $P(\kappa)$ emerging from the non-linear growth prior to recombination, we will follow the approach outlined in Melia (2014) and parametrize it as follows,

$$
P(\kappa) \propto \kappa-b\left(\frac{2 \pi}{R_{e}\left(t_{e}\right)}\right)^{2} \kappa^{-1},
$$

where the (unknown) constant $b$ is expected to be $\sim O(1)$.

This form of $P(\kappa)$ is based on the following reasoning. One typically assumes a scale-free initial power-law spectrum, which is what one might have expected with or without the complex machinery of inflation. Such a spectrum is often referred to as a Harrison-Zeldovich-Peebles spectrum, since these were the indivdiuals who first proposed it as appropriate for the initial conditions many years prior to the development of inflationary models. (In fact, a better signature of inflation is a small deviation from a scale-free spectrum and/or a mixture of tensor modes.) In $\Lambda$ CDM, these fluctuations grow and then expand on all scales during the required inflationary phase. In $R_{\mathrm{h}}=c t$, the fluctuation growth is driven by the (negative) pressure, represented by the term on the right-hand side of equation (10). Because there is no Jeans length, fluctuations can in principle grow on all scales as well. However, this equation also shows that what matters most is the ratio of the fluctuation 
length $\lambda$ to the gravitational radius $R_{\mathrm{h}}(t)$ at time $t$. The solution to this equation shows that only fluctuations with $\lambda<2 \pi R_{\mathrm{h}}$ will grow, and that those modes that grow, will grow rapidly, given their strong dependence on $t$ (see equation 16).

One can see from this equation and the definition of $P(\kappa)$ that for $\lambda<<2 \pi R_{\mathrm{h}}$, corresponding to large $\kappa$, we have $\alpha \sim \kappa_{0} \kappa$, where $\kappa_{0} \equiv c t_{0} / \sqrt{3}$, so that

$$
P(\kappa) \approx \kappa t^{2 \kappa_{0} \kappa} \quad(\text { large } \kappa)
$$

On the other hand, for $\lambda>>2 \pi R_{\mathrm{h}}$, corresponding to small $\kappa$, we have $\alpha \sim\left(\kappa_{0} \kappa\right)^{2} / 2$, and so

$$
P(\kappa) \approx \kappa t^{\left(\kappa_{0} \kappa\right)^{2}} \quad(\text { small } \kappa)
$$

For a given value of the cosmic time (say at recombination, $t_{\mathrm{e}}$ ), we can express this changing behavior for small and large $\kappa$ as a sum of polynomials,

$$
P(\kappa)=\sum_{i} c_{i} \kappa^{i}+\sum_{j} b_{j} \kappa^{-j},
$$

with leading order terms $P(\kappa) \sim c_{1} \kappa+b_{1} \kappa^{-1}$. The choice of $c_{1}$ and $b_{1}$ shown in equation (25) provides a reasonable representation of the evolution with $\kappa$ from equation(26) to $(27)$.

What this means physically is that the fluctuations will grow quickly in amplitude up to the size $2 \pi R_{\mathrm{h}}(t)$, above which the growth is suppressed. The simple parametrization in equation (25) incorporates these essential effects: first, the initial seed spectrum is assumed to be scale-free, which means that $P(\kappa) \sim \kappa$. Since the growth rate depends critically on the ratio $R_{\mathrm{h}} / \lambda$, one would expect $P(\kappa)$ to be dominated by the smaller wavelengths (i.e., the larger $\kappa$ 's), and be altered more and more for increasing wavelengths (i.e., smallter $\kappa$ 's). Since the growth rate decreases with decreasing $\kappa$, one would expect a greater 
and greater depletion in power. The second term in equation (25) represents this effect.

Now, from equations (24) and (25), we see that

$$
\left(\frac{\delta \rho}{\rho}\right)_{\lambda}^{2} \propto \frac{1}{4} \kappa^{4}-\frac{1}{2} b\left(\frac{2 \pi}{R_{e}\left(t_{e}\right)}\right)^{2} \kappa^{2} .
$$

Defining the angle $\theta$ analogously with $\theta_{\max }$ in equation (20), we put

$$
\theta \equiv \frac{\lambda}{R_{e}\left(t_{e}\right)}
$$

whereupon

$$
\left(\frac{\delta \rho}{\rho}\right)_{\lambda}^{2} \propto \frac{1}{4} \kappa^{4}\left(1-2 b \theta^{2}\right) .
$$

Therefore, keeping only higher order terms in the binomial expansion for the square root of the factor on the right, we find that

$$
\delta \rho \sim \frac{1}{\lambda^{2}}\left(1-b \theta^{2}\right)
$$

Thus, the amplitude of the Sachs-Wolfe temperature fluctuations follows the very simple form

$$
\frac{\Delta T}{T} \sim\left(1-b \theta^{2}\right)
$$

but only up to the maximum angle $\theta_{\max }$ established earlier.

In comparing the angular correlation function $C(\theta)$ resulting from this expression with that inferred from the WMAP data, we found earlier that the general shape of $C(\theta)$ depends only weakly on the chosen values of $b$ and $t_{0} / t_{e}$. Insofar as the large-scale fluctuations are concerned, therefore, the principal feature of the $R_{\mathrm{h}}=c t$ Universe that distinguishes it from $\Lambda \mathrm{CDM}$ is the existence of the maximum angle $\theta_{\max }$. We anticipate that the outcome will be similar here if the two large-scale anomalies are indeed linked in this cosmology. 


\section{The CMB Power Spectrum for Low- $l$}

Throughout our discussion in this paper, we focus solely on fluctuations induced by the Sachs-Wolfe effect, ignoring other important physical processes, such as Baryon Accoustic Oscillations, that dominate on scales of a few degrees, or less. Since the values of $b$ and $t_{0} / t_{e}$ were essentially identified from our study of the angular correlation function, the principal unknown here is therefore the number $N_{S W}$ of Sachs-Wolfe fluctuations across the sky. The CMB power spectrum at angles $>>1^{\circ}$ arises from these, so it should be possible to fit the data for $l<20$ using the $C_{l}$ 's calculated from Equations (1)-(5) and (27), in order to infer the approximate range of values of $N_{S W}$ implied by the observations.

The CMB power spectrum is calculated according to

$$
\delta T_{l}^{2}=l(l+1) C_{l} / 2 \pi
$$

with the angular power $C_{l}$ of multipole $l$ given in Equation (5). Several theoretical curves showing $\delta T_{l}$ versus $l$ are shown in Figures $1-4$, for various choices of the 2 principal parameters at our disposal, the constant $b$ in Equation (27), and $N_{S W}$. The CMB's angular correlation function also depends quantitatively (though not qualitatively) on the ratio $t_{0} / t_{e}$, which defines $\theta_{\max }$ in Equation (22) (Melia 2014). Eventually, detailed simulations of the fluctutations growth with the timeline afforded by the $R_{\mathrm{h}}=c t$ Universe will provide a reliable estimate of this ratio, so we won't have to treat it as an unknown parameter. But for now, the results of the angular-correlation function analysis indicate that the effects due to an increase in $b$ can be offset by an increase in $t_{0} / t_{e}$. This degeneracy, however, does not carry over to the process of low- $l$ multiple alignment, since the results here do not appear to be sensitive to the ratio $t_{0} / t_{e}$. So we will use the same value $\left(t_{0} / t_{e}=5 \times 10^{3}\right)$ in every simulation. By way of interest, we note that a redshift of $\sim 1100$ (associated with the surface of last scattering in $\Lambda \mathrm{CDM})$ corresponds to a ratio $t_{0} / t_{e}=(1+z) \sim 10^{3}$ in $R_{\mathrm{h}}=c t$. As is the case in the standard model, the absolute scale is not known a priori, so 


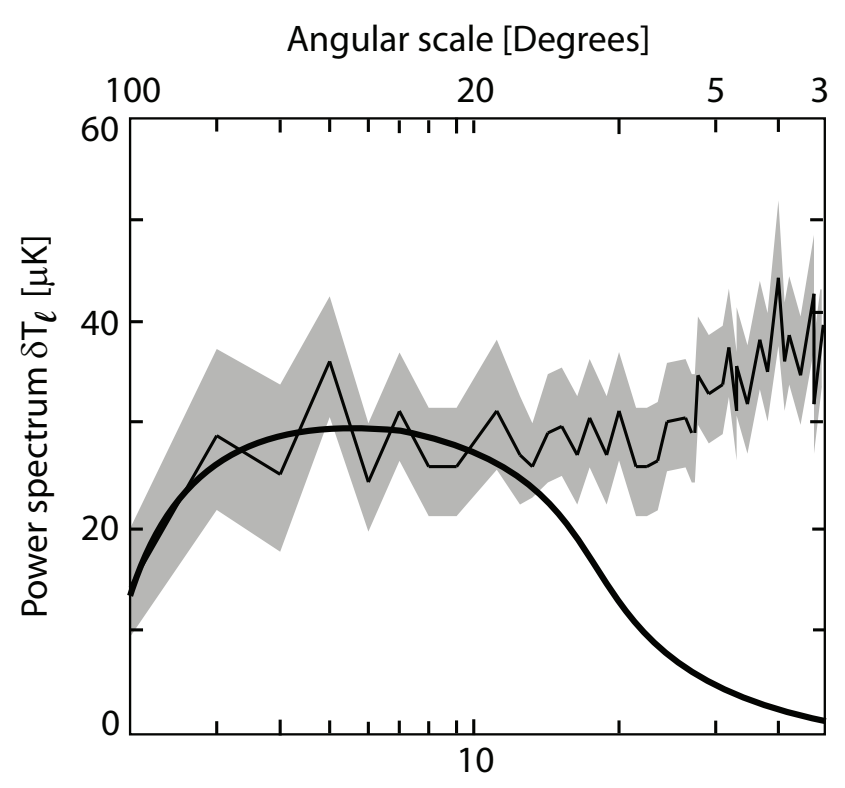

Multipole $\ell$

Fig. 1. - The theoretical CMB power spectrum due solely to Sachs-Wolfe-induced fluctuations in the $R_{\mathrm{h}}=c t$ Universe (solid, thick curve), in comparison with the power spectrum measured from the full WMAP sky (thin, jagged line; Spergel et al. 2003; Tegmark et al. 2003). The gray region represents the one- $\sigma$ uncertainty. The number of fluctuations assumed for this simulation is $N_{S W}=5,000$. The power spectrum for $l>20$ is dominated by small-scale physical effects, such as Baryon Acoustic Oscillations near the surface of last scattering, which are not included in our analysis. See text for other details.

the amplitude is also adjustable, e.g., by fitting $\delta T_{5}$ to the data.

The theoretical curve shown in Figure 1 was calculated using $b=12$ and $N_{S W}=5,000$, which fits the WMAP power spectrum quite well for $l<20$, corresponding to angular scales $>10^{\circ}$. Not surprisingly, the Sachs-Wolfe fluctuations produce very little power on scales smaller than this, where more localized effects (e.g., near the surface of last scattering) dominate the perturbation growth. We note, in particular, that the power spectrum predicted by the $R_{\mathrm{h}}=c t$ Universe at $l \rightarrow 1$ agrees with the relative lack of power observed 


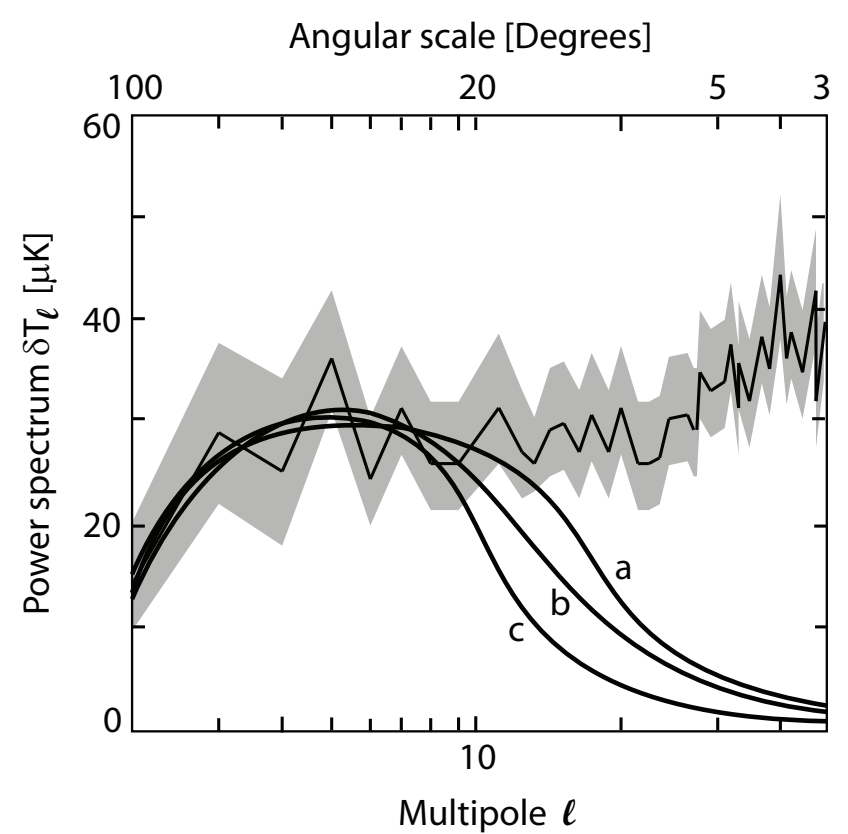

Fig. 2.- Comparison of theoretical CMB power spectra due solely to Sachs-Wolfe-induced fluctuations for $N_{S W}=5,000$ and various values of $b$ : a) (same as figure 1) $b=12$, b) $b=8$, and c) $b=4$. The gray region represents the one- $\sigma$ uncertainty.

for the low- $l$ multipoles, a result we had previously discussed in the context of the CMB angular correlation function (Melia 2014). Planck has recently confirmed the surprising WMAP observation of a lack of correlation at angles $>60^{\circ}$ (Ade et al. 2013), which does not appear to be consistent with an inflationary scenario. As we discussed previously, the downturn in power towards small values of $l$ in the $R_{\mathrm{h}}=c t$ Universe is entirely due to the maximum angular size $\theta_{\max }$ of fluctuations expected in this cosmology (see Equation 22).

A comparison of curves in Figures 2-4 demonstrates how these results depend on $b$ and $N_{S W}$. We see in Figure 2 that the spectrum below $l \sim 10$ is rather insensitive to the precise value of $b$. Eventually, when other small-scale physical processes are included, it may be possible to more tightly constrain $b$ based on a comparison of the spectrum with the data at $l \sim 10-30$. Figure 3 shows that fluctuation numbers $N_{S W}$ much greater than 5,000 (in 


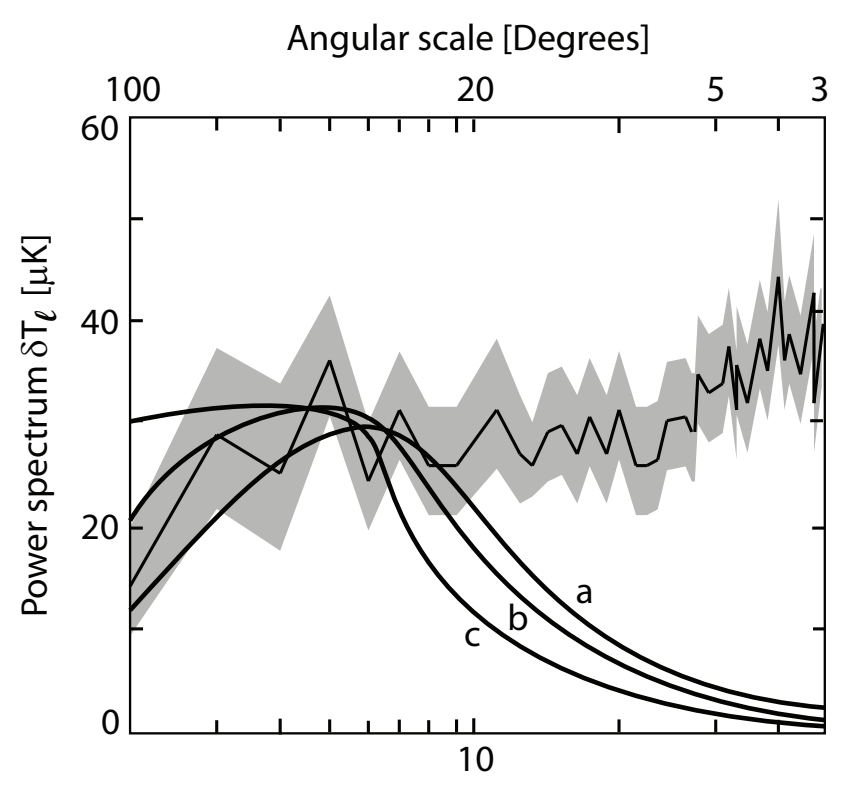

Multipole $\ell$

Fig. 3. - Same as Figure 2, except here for $N_{S W}=30,000$. The curves correspond to a) $b=12$, b) $b=8$, and c) $b=4$. The gray region represents the one- $\sigma$ uncertainty.

this case, 30,000) do not produce a power spectrum matching the CMB's observed features at $l<10$. These spectra are either too sharply peaked at $l \sim 5-6$ or, in the case of c), show too much power at $l<5$. And in figure 4 , we see that a value of $N_{S W}$ as low as 1,000 may work with a relatively high value of $b$, i.e., $b \sim 12$, corresponding to curve a), but probably not for smaller values of this parameter, which seem to produce power spectra that are too sharply peaked at $l \sim 5$.

All in all, this brief survey of the available range of $N_{S W}$ and $b$ shows that the number $N_{S W}$ of Sachs-Wolfe fluctuations greater than $\sim 5,000$ is unlikely to fit the observed CMB power spectrum at large angles, while a number $<1,000$ produces a power distribution too highly peaked at $l \sim 2-5$. These simulations suggest that in the $R_{\mathrm{h}}=c t$ Universe, $N_{S W}$ is several thousand, and probably no bigger than $\sim 5,000$. 


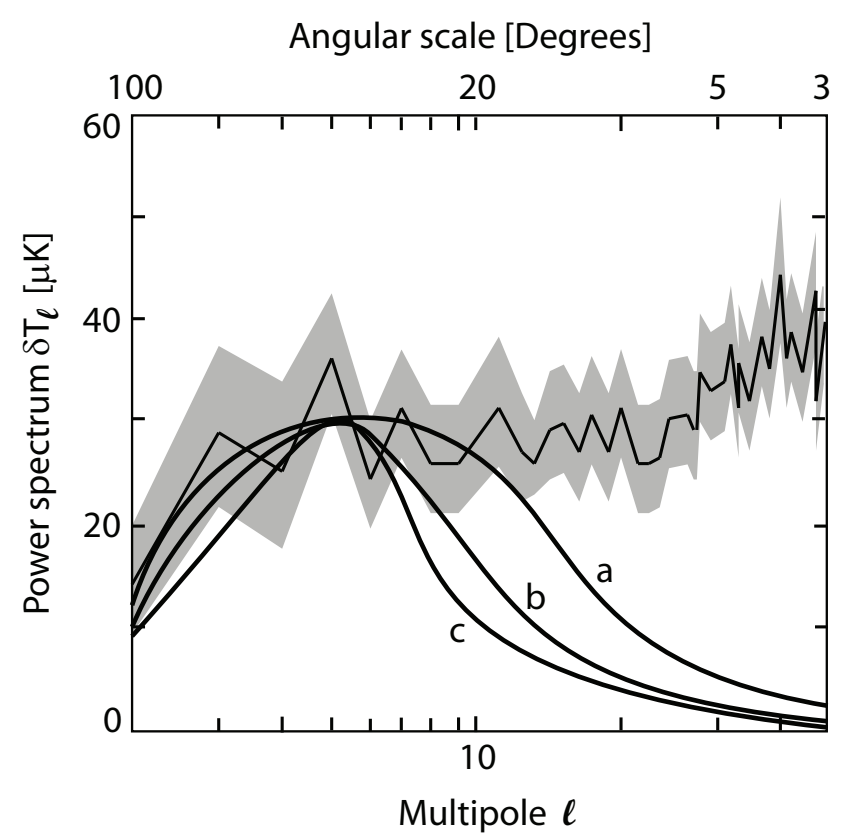

Fig. 4.- Same as Figure 2, except here for $N_{S W}=1,000$. The curves correspond to (a) $b=12$, (b) $b=8$, and (c) $b=4$. The gray region represents the one- $\sigma$ uncertainty.

\section{The CMB Quadrupole and Octopole Moments}

In Figure 5, we show three simulated renderings of the large-scale fluctuations in the CMB temperature using values of $b(\sim 3)$ and $t_{0} / t_{e}\left(\sim 5 \times 10^{3}\right)$ indicated by our earlier fits to the angular correlation function, and $N_{S W}=1,000$. We emphasize again that none of the effects thought to produce fluctuations on $<1^{\circ}$ scales, such as acoustic oscillations and the various processes producing secondary signatures after decoupling, are included in these images (see Melia 2014, and references cited therein, for a more complete discussion of all the relevant physical mechanisms). Previous work has shown that these other processes are not directly relevant to the $l=2$ and $l=3$ multipole moments. The principal features evident in this figure are due solely to the Sachs-Wolfe effect, but strictly adhering to the restrictions imposed by Equations (21) and (26).

Before entering into a quantitative statistical analysis of these simulated all-sky maps, 

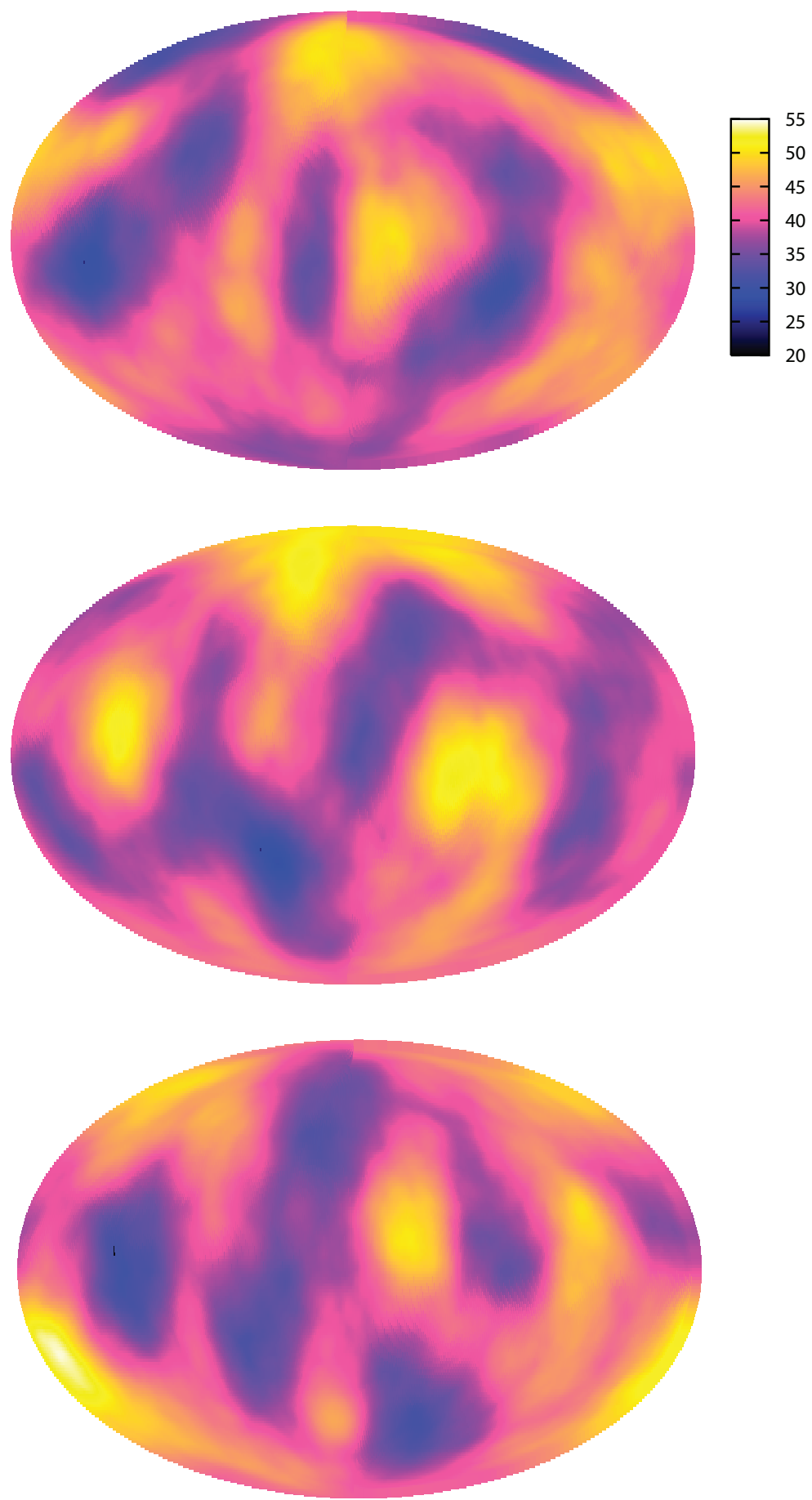

Fig. 5.- Three simulated renderings of the large-scale fluctuations in the CMB temperature for the $R_{\mathrm{h}}=c t$ Universe. Here, $t_{0} / t_{e}=5 \times 10^{3}$ and $b=3$. Each image contains $N_{S W}=1,000$ large-size fluctuations. The units on the color scale are arbitrary. 
it is quite evident even by eye that the general features emerging from the $R_{\mathrm{h}}=c t$ Universe are reminiscent of those actually seen in the WMAP data. Note in particular the apparent "planarity" of the fluctuations, and the emergence of "finger-like" darker regions. The apparent planar-like arrangement of the octopole components was first noted by de Oliveira et al. (2004), but revisited by many authors since then. In the analysis of Park et al. (2007), the probability of observing such a planarity within the context of the standard model is over $18 \%$, and therefore not statistically significant. It is comforting from the standpoint of the $R_{\mathrm{h}}=c t$ Universe that this feature appears to be present most of the time. The finger-like depressions are more difficult to quantify, but were noted by Bennett et al. (2011). Again, it is apparent from these simulations that such features are rather common in the $R_{\mathrm{h}}=c t$ Universe.

Let us now examine how much impact the restricted range of fluctuation angles $\left(\theta<\theta_{\max }\right)$ has on the distribution of $\theta_{23}$. We have produced 20,000 simulated all-sky CMB maps for each assumed value of $N_{S W}$, ranging from 5 to 5,000. As noted earlier, a sample of these for $N_{S W}=1,000$ is shown in figure 5 .

Of course, the number $N_{S W}$ of Sachs-Wolfe-induced fluctuations cannot be predicted from theory. Only their growth rate is quantifiable using equations (11) to (17). For this reason, we must rely on the outcome not having a strong dependence on this parameter, which we therefore vary over such a broad range of values in order to ensure that the statistical properties associated with the quadrupole and octopole orientations are not overly influenced by it. The results will show that the principal reason for the differences between $\Lambda \mathrm{CDM}$ and $R_{\mathrm{h}}=c t$ is in fact the maximum size of the fluctuations, set by the gravitational horizon at the time of last scattering (equations 18-22).

For each synthetic map, we followed the procedure outlined in $\S 2$ above, using the various techniques described in Appendix A of de Oliveira et al. (2004) to find the $a_{l m}(\hat{\mathbf{n}})$ 
coefficients in the rotated frame. From these, we calculated the values of $\theta_{23}$ (between the quadrupole and octopole moments) and determined their occurrence rate. The corresponding relative probabilities are shown in Figure 6, for $N_{S W}=5,20,50$, and 5,000.
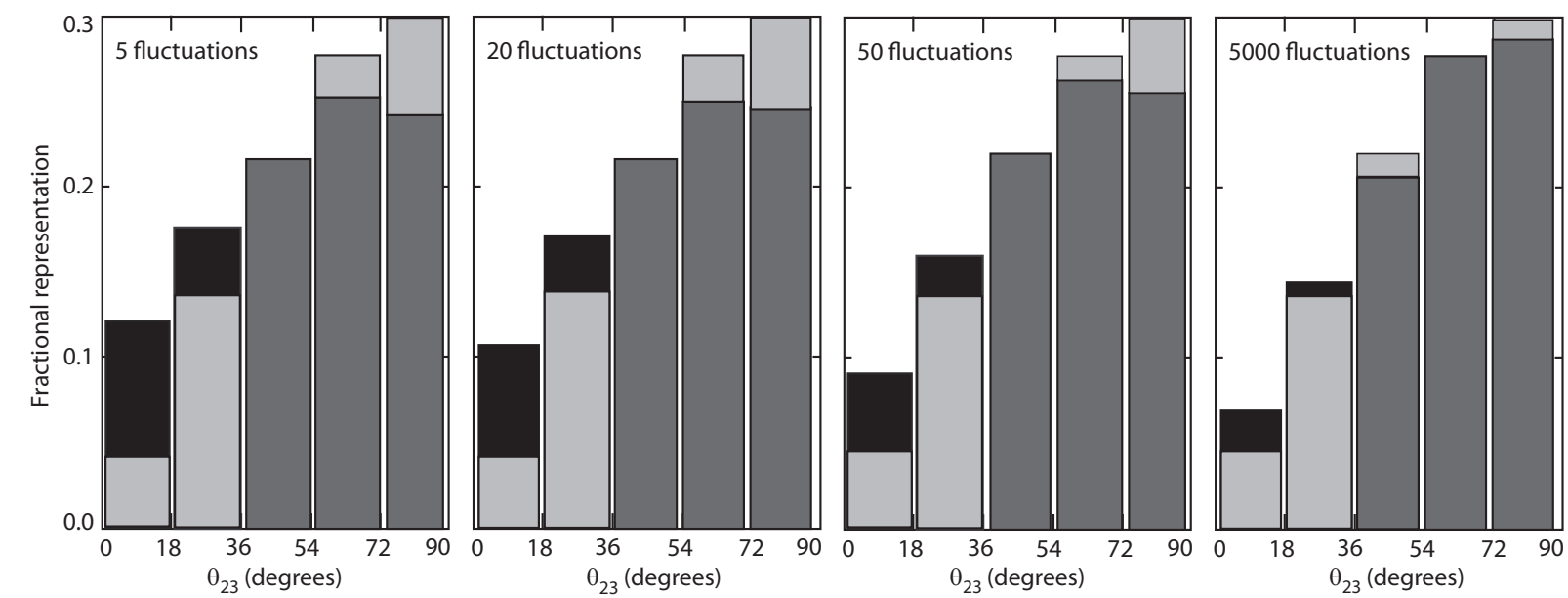

Fig. 6. - Fractional representation of the angle $\theta_{23}$ between the CMB quadrupole and octopole moments in 20,000 simulated renderings of the $R_{\mathrm{h}}=c t$ Universe, assuming a total number of 5, 20, 50, and 5,000 large-size fluctuations, respectively (left to right). The light-grey bars show the fractions in a $\Lambda$ CDM universe with fluctuations sampled from a statistically isotropic, Gaussian random field of zero mean. The black and dark-grey bars show the corresponding fractions for the $R_{\mathrm{h}}=c t$ Universe.

Mindful of the conclusions drawn by Park et al. (2007), in which the observed alignment angle appears to fall within the range $3.8^{\circ}<\theta_{23}<18.2^{\circ}$, we have chosen to subdivide the results into increments of $18^{\circ}$, so that the most likely value of the measured $\theta_{23}$ lies within the first bin. The probability distribution for a completely random occurrence of the angle $\theta_{23}$ corresponds to the light-grey bars in these diagrams, essentially the profile expected in the standard model. The probability of alignment within the first bin is the aforementioned value of $4.9 \%$, so the observed angle $\theta_{23}$ constitutes a marginally statistically significant anomaly. In contrast, the probabilities expected for the $R_{\mathrm{h}}=c t$ Universe correspond to the 
black and dark-grey bars.

Notice that the overall probability distribution depends on how many fluctuations are included in the simulation. As $N_{S W}$ increases to very large values, we approach the result expected for $\Lambda \mathrm{CDM}$, presumably because this situation is similar to what one gets with a fluctuation spectrum inflated to very large scales early in the Universe's history. Absent inflation, however, the probability distribution is noticeably different, particularly for the smaller values of $\theta_{23}$. For $N_{S W}=50$, a value of $\theta_{23}<18^{\circ}$ is expected to occur about $10 \%$ of the time-higher for smaller fluctuation numbers $N_{S W}$. And for $N_{S W}=5,000$, this probability is approximately $7 \%$. In every case, the increase in fractional representation at smaller angles is compensated by the reduced representation at angles $>50^{\circ}$ (the dark-grey bars in these diagrams). These results suggest that an alignment of the quadrupole and octopole moments to within $\sim 18^{\circ}$ of each other is more likely to occur in the $R_{\mathrm{h}}=c t$ Universe than in $\Lambda \mathrm{CDM}$, as long as the number of Sachs-Wolfe fluctuations across the sky is smaller than several thousand, though in both cosmologies, the probability is always $\leq 10 \%$.

\section{Discussion and Conclusions}

It was shown in a detailed analysis of the CMB large-scale anomalies by Sarkar et al. (2011) that there is no statistically significant correlation in $\Lambda$ CDM between the missing power on large angular scales and the alignment of the $l=2$ and $l=3$ multipoles. If the CMB anomalies are not due to astrophysical, instrumental, or data analysis effects, but instead arise from cosmological influences in the early Universe, and if we ignore possible biases introduced by the posterior selection of these two particular features in the CMB, then the tension between the standard model and the WMAP data is greater than for each anomaly alone, because their combined statistical significance could be as 
small as the product of their individual significances.

As we have noted in the introduction, there are good reasons to believe that many effects may be responsible for the observed anomalies, so it is not clear that one should place too much confidence on how these features impact the models themselves. In particular, one should take note of the fact that when the contribution of the ISW effect to the anisotropy pattern is removed from the WMAP map, the statistical significance of the apparent quadrupole-octopole alignment decreases considerably (Francis and Peacock 2010; Bennett et al. 2011). If this is the dominant cause of the alignment, then clearly it has very little to do with physics in the early Universe.

In this paper, we have addressed the question of whether the observed alignment may in fact be due to cosmological influences, and if so, whether its properties may be used to discriminate between competing models. We have sought to calculate the probability of seeing this feature in the CMB anisotropies for the $R_{\mathrm{h}}=c t$ Universe. Earlier, we had demonstrated that the angular correlation function in this cosmology apparently agrees with the WMAP and Planck observations without the need to invoke cosmic variance. As such, the overall probability of seeing no power on large angular scales and an apparent alignment of the low- $l$ multipoles is due predominantly to the latter. Though not directly related, these two features of the CMB nonetheless have a common origin in the $R_{\mathrm{h}}=c t$ Universe - the existence of a maximum angular size $\theta_{\max }$ for the large-scale fluctuations, imposed by the gravitational horizon $R_{\mathrm{h}}$ at the time $t_{e}$ of last scattering. Our conclusion from this work is that in $R_{\mathrm{h}}=c t$, the simultaneous observation of the missing large-angle correlations and low- $l$ multipole alignment is likely at the $7-10 \%$ level, depending on what the actual number of Sachs-Wolfe fluctuations $N_{S W}$ turns out to be. It is also useful to point out that the increase in the probability of alignment expected in the $R_{\mathrm{h}}=c t$ Universe for $C_{i}$ and $C_{i+1}$ drops rapidly to levels comparable to those 
in $\Lambda \mathrm{CDM}$ for $i>2$. For example, the fractional representation for $\theta_{34}$ using $\mathbf{5 , 0 0 0} N_{S W}$ fluctuations (see figure 6 ) is already $\sim 5 \%$, comparable to the value $(\sim 4.9 \%)$ in $\Lambda$ CDM. The probability for higher values of $i$ is indistinguishable from a completely random occurrence.

Aside from the quantitative aspects of this analysis, a qualitative comparison between the simulated sky maps shown in Figure 5, and the real Universe as revealed by WMAP and Planck, also suggests a morphological similarity between the two. We noted the appearance of "finger-like" darkened extensions and the planarity of the octopole components which, however, are not statistically significant, even in the standard model. Overall, the weight of evidence - the angular correlation function, the smaller statistical significance of the alignment of the quadrupole and octopole moments, and the morphological similarity between the real and simulated CMB maps - seems to favor the $R_{\mathrm{h}}=$ ct Universe over $\Lambda \mathrm{CDM}$, if these features are all due to the cosmology itself rather than to the many other possible causes proposed since the observations.

Clearly, there is still work to do. By necessity, our analysis of the angular correlation function and the low-multipole alignment has relied on a highly simplified treatment of the fluctuation growth in the early Universe. It is well known, however, that there are many mechanisms producing density perturbations, on small and large scales, and there is a great deal of astrophysics linking these to the actual temperature variations we see across the sky. Our approach here has merely shown promise in accounting for the observations. We cannot be completely certain of the outcome until we have developed a more sophisticated treatment of the fluctuation growth in the $R_{\mathrm{h}}=c t$ Universe, commensurate with the level of detail already incorporated into the standard model.

I am grateful to the anonymous referee for his careful review of this paper and for recommending several important improvements to its content. This 
research was partially supported by ONR grant N00014-09-C-0032 at the University of Arizona, and by a Miegunyah Fellowship at the University of Melbourne. I am particularly grateful to Amherst College for its support through a John Woodruff Simpson Lectureship. 


\section{REFERENCES}

Ade, P.A.R. et al. 2013, A\&A, in press (arXiv:1303.5083)

Bennett, C. L. et al., 2003, ApJS, 148, 97

Bennett, C. L. et al., 2011, ApJS, 192, Article ID 17

Bielewicz, P., Eriksen, H. K., Banday, A. J., Gorski, K. M. and Liljie, P. B. 2005, ApJ, 635, 750

Chiang, L.-Y., Naselsky, P. D. and COles, P. 2007, ApJ, 664, 8

Cooray, A. and Seto, N. 2005, JCAP, 0512, 004

Copi, C. J., Huterer, D. and Starkman, G. D. 2004, Phys. Rev. D, 70, 043515

Copi, C. J., Huterer, D., Schwarz, D. J. \& Starkman, G. D., 2009, MNRAS, 367, 79

Copi, C. J., Huterer, D., Schwarz, D. J. \& Starkman, G. D., 2009, MNRAS, 399, 295

Copi, C. J., Huterer, D., Schwarz, D. J. \& Starkman, G. d., 2010, Adv. Astr., 2010, id. 847541

de Oliveira-Costa, A., Tegmark, M., Zaldarriage, M. \& Hamilton, A., 2004, Phys. Rev. D, 69, Article ID 063516

Dikarev, V., Preuss, O., Solanki, S. Kruger, H and Krivov, A. 2008, Earth Moon and Planets, 102, 555

Dikarev, V., Preuss, O., Solanki, S. Kruger, H and Krivov, A. 2009, ApJ, 705, 670

Efstathiou, G., 1990, in Physics of the Early Universe, eds. J. A. Peacock, A. F. Heavens \& A. T. Davies, SUSSP, Edinburgh 
Efstathiou, G., Ma, Y. and Hanson, D. 2010, MNRAS, 407, 2530

Eriksen, H. K., Hansen, F. K., Barday, A. J., Górski, K. M. \& Lilje, P. B., 2004, ApJ, 605, 14

Francis, C. L. and Peacock, J. A. 2010, MNRAS, 406, 14

Frisch, P. C. 2005, ApJ Lett., 632, L143

Gordon, C., Hu, W., Huterer, D. and Crawford, T. M. 2005, Phys. Rev. D, 72, 103002

Guth, A. H., 1981, Phys. Rev. D, 23, 347

Hansen, F. K., Cabella, P., Marinucci, D. \& Vittorio, N., 2004, ApJ Letters, 607, L25

Hinshaw, G. et al., 1996, ApJ Letters, 464, L25

Inoue, K. T. and Silk, J. 2006, ApJ, 648, 23

Land, K. \& Magueijo, J., 2005, PRL, 95, Article ID 071301

Linde, A., 1982, Phys. Lett. B, 108, 389

Melia, F., 2007, MNRAS, 382, 1917

Melia, F., 2012a, Australian Phys., 49, 83

Melia, F., 2012b, AJ, 144, 110

Melia, F. 2013a, A\&A, 553, id.A76

Melia, F., 2013b, ApJ, 764, 72

Melia, F., 2014, A\& A, 561, id.A80

Melia F. \& Maier, R., 2013, MNRAS, 432, 2669 
Melia, F. \& Shevchuk, A., 2012, MNRAS, 419, 2579

Park, C.-G., Park, C. \& Gott, J. R., 2007, ApJ, 660, 959

Peiris, H. V. and Smith, T. L. 2010, Phys. Rev. D, 81, 123517

Rakic, A., Rasanen, S. and Schwarz, D. J. 2006, MNRAS, 369, L27

Rakic, A. and Schwarz, D. J. 2007, Phys. Rev. D, 75, 103002

Sachs, R. K. \& Wolfe, A. M., 1967, ApJ, 147, 73

Sarkar, D., Huterer, D., Copi, C. J., Starkman, G. D. \& Schwarz, D. J., 2011, Astroparticle Phys., 34, 591

Schwarz, D. J., Starkman, G. D., Huterer, D. \& Copi, C. J., 2004, PRL, 93, Article ID 221301

Slosar, A. and Seljak, U. 2004, Phys. Rev. D, 70, 083002

Spergel, D. N. et al., 2003, ApJS, 148, 175

Tegmark, M., de Oliveira-Costa, A. \& Hamilton, A. J., 2003, Phys. Rev. D, 68, Article ID 123523

Vale, C. 2005, arXiv:astro-ph/0509039

Wei, J.-J., Wu, X. \& Melia, F., 2013, ApJ, 772, 43

Weyl, H. 1923, Z. Phys., 24, 230

Wright, E. L., Bennett, C. L., Gorski, K., Hinshaw, G. \& Smoot, G. F., 1996, ApJ Letters, 464, L21 